\title{
Alpha Power Transformed Inverse Lomax Distribution with Different Methods of Estimation and Applications
}

\author{
Ramadan A. ZeinEldin, ${ }^{1,2}$ Muhammad Ahsan ul Haq ${ }^{(D)}{ }^{3,4}$ Sharqa Hashmi ${ }^{\text {D, }},{ }^{4,5}$ \\ and Mahmoud Elsehety (iD $^{6}$ \\ ${ }^{1}$ Deanship of Scientific Research, King Abdulaziz University, Jeddah, Saudi Arabia \\ ${ }^{2}$ Faculty of Graduate Studies for Statistical Research, Cairo University, Giza, Egypt \\ ${ }^{3}$ Quality Enhancement Cell, National College of Arts, Lahore, Pakistan \\ ${ }^{4}$ College of Statistical \& Actuarial Sciences, University of the Punjab, Lahore, Pakistan \\ ${ }^{5}$ Lahore College for Women University (LCWU), Lahore, Pakistan \\ ${ }^{6}$ King Abdulaziz University, Jeddah, Saudi Arabia \\ Correspondence should be addressed to Muhammad Ahsan ul Haq; ahsanshani36@gmail.com
}

Received 10 October 2019; Revised 2 January 2020; Accepted 20 January 2020; Published 27 February 2020

Academic Editor: Quanmin Zhu

Copyright (c 2020 Ramadan A. ZeinEldin et al. This is an open access article distributed under the Creative Commons Attribution License, which permits unrestricted use, distribution, and reproduction in any medium, provided the original work is properly cited.

In this paper, a new three-parameter lifetime distribution, alpha power transformed inverse Lomax (APTIL) distribution, is proposed. The APTIL distribution is more flexible than inverse Lomax distribution. We derived some mathematical properties including moments, moment generating function, quantile function, mode, stress strength reliability, and order statistics. Characterization related to hazard rate function is also derived. The model parameters are estimated using eight estimation methods including maximum likelihood, least squares, weighted least squares, percentile, Cramer-von Mises, maximum product of spacing, Anderson-Darling, and right-tail Anderson-Darling. Numerical results are calculated to compare the performance of these estimation methods. Finally, we used three real-life datasets to show the flexibility of the APTIL distribution.

\section{Introduction}

The inverse Lomax (IL) is originally developed as a lifetime distribution. The inverse Lomax is member of family of generalized beta distribution. Kleiber and Kotz [1] showed that IL distribution can be used in economics. The IL distribution has many applications in modeling of different trends of hazard rate function (hrf), i.e., decreasing or upside-down bathtub failure rate of life testing of components. The IL distribution is used in [2] to get Lorenz ordering relationship among ordered statistics. McKenzie et al. [3] used the IL model and applied it to geophysical databases. Singh et al. [4] investigated the reliability estimators of IL distribution under Type II censoring. Bayesian estimation of mixture of the IL model under the censoring scheme was studied in [5]. Inverse power Lomax distribution was studied in [6] and Weibull IL distribution in [7].

The probability density (pdf) and cumulative distribution function (cdf) of the IL distribution are as follows:

$$
\begin{aligned}
& g(x ; a, b)=\frac{a b}{x^{2}}\left(1+\frac{b}{x}\right)^{-a-1}, \quad x, a, b>0, \\
& G(x ; a, b)=\left(1+\frac{b}{x}\right)^{-\alpha}, \quad x, a, b>0 .
\end{aligned}
$$

Mahdavi and Kundu [8] introduced the alpha power transformation (APT) method to add an additional parameter to a family of distributions to increase flexibility in given family. The cdf and pdf of APT-G family are 


$$
F(x ; \alpha)=\left[\begin{array}{ll}
\frac{\alpha^{G(x)}-1}{\alpha-1}, & \text { if } \alpha>0, \alpha \neq 1, \\
G(x), & \text { if } \alpha=1,
\end{array}\right.
$$

and the corresponding pdf is

$$
f(x ; \alpha)=\left[\begin{array}{ll}
\frac{\log (\alpha)}{\alpha-1} g(x) \alpha^{G(x)}, & \text { if } \alpha>0, \alpha \neq 1, \\
g(x), & \text { if } \alpha=1 .
\end{array}\right.
$$

In the literature, many probability distributions are generalized using this approach; for example, alpha power transformed Weibull (APTW) distribution in [9], APT generalized exponential distribution in [10], APT Lindley distribution in [11], APT extended exponential distribution in [12], alpha power inverted exponential distribution in [13], alpha power Inverse-Weibull distribution in [14], APT inverse-Lindley distribution in [15], APT power Lindley studied in [16], and APT Pareto distribution proposed in [17].

The main goal of this research article is to introduce a simpler and more flexible model called APT inverse Lomax (APTIL) distribution. Furthermore, the key motivations of using APTIL distribution in the practice are follows:

(i) To improve the flexibility of the existing distributions by using APT-G (ii) To introduce the extended version of the IL distribution whose closed form of cdf exist.

(iii) To provide better fits than the competing modified models

The rest of the paper is arranged as follows. In Section 2, we define the new model called AP inverse Lomax (APTIL) distribution. Various statistical properties of the APTIL distribution are derived in Section 3 along with more attractive expressions for quantile function, median, mode, moments, order statistics, and stress strength parameter. Lemmas 1 and 2 contain expressions for stochastic ordering and characterization related to hazard function, and the asymptotic behavior of density is derived. In Section 4, we estimate the parameters using eight methods of parameters estimation as follows: maximum likelihood (ML), least squares (LS), weighted least squares (WLS), percentile (PC), Cramer-von Mises (CV), maximum product of spacing (MPS), Anderson-Darling (AD), and right-tail Anderson-Darling (RTAD). Section 5 deals with three applications to show the efficiency of the proposed model. Final remarks are mentioned in Section 6.

\section{APTIL Distribution}

The random variable (rv) $X$ is said to have APTIL distribution denoted by $\operatorname{APTIL}(\alpha, a$, and $b)$ with two shape parameters and one scale as $\alpha, a$, and $b$, respectively. The pdf of $X$ for $x \geq 0$ is

$$
\begin{aligned}
& f(x)= \begin{cases}\frac{\log (\alpha)}{\alpha-1} \frac{a b}{x^{2}}\left(1+\frac{b}{x}\right)^{-a-1} \alpha^{(1+(b / x))^{-a}}, & \text { if } \alpha \neq 1, a, b, \alpha>0, \\
\frac{a b}{x^{2}}\left(1+\frac{b}{x}\right)^{-a-1}, & \text { if } \alpha=1, a, b, \alpha>0,\end{cases} \\
& F(x)= \begin{cases}\frac{\alpha^{(1+(b / x))^{-a}}-1}{\alpha-1}, & \text { if } \alpha \neq 1, \alpha>0, \\
\left(1+\frac{b}{x}\right)^{-a}, & \text { if } \alpha=1, \alpha>0 .\end{cases}
\end{aligned}
$$

The survival function (sf) and the hrf for APTIL distribution for $x>0$ are in the following forms:

$$
\begin{aligned}
& S(x)=\frac{\alpha-\alpha^{(1+b / x)^{-a}}}{\alpha-1}, \\
& h(x)=\frac{\log (\alpha) a b(1+(b / x))^{-a-1} \alpha^{(1+(b / x))^{-a}}}{x^{2}(\alpha-1)\left\{1-\alpha^{(1+(b / x))^{-a}}-1 / \alpha-1\right\}} .
\end{aligned}
$$

Figure 1 demonstrates the graphs of pdf and hazard function of APTIL distribution for different values of $\alpha, a$, and $b$. Clearly, the pdf of APTIL distribution is the function for $\alpha \neq 1$ and $a<1$ and unimodal for $\alpha \neq 1$ and $a>1$. The hrf of the APTIL model can be decreasing or upside-down bathtub for $\alpha \neq 1$ and $a<1$ and $a>1$, respectively.

2.1. Useful Expansions. Here, an explicit expression for APTIL pdf is given. By using the series representation of exponential function, equation (5) can be written as 


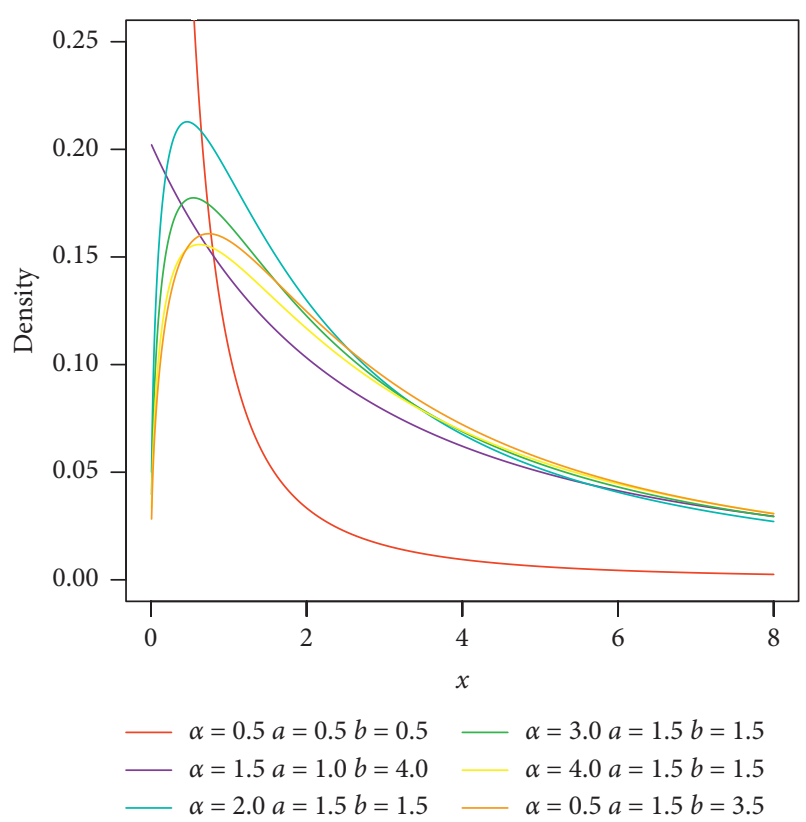

(a)

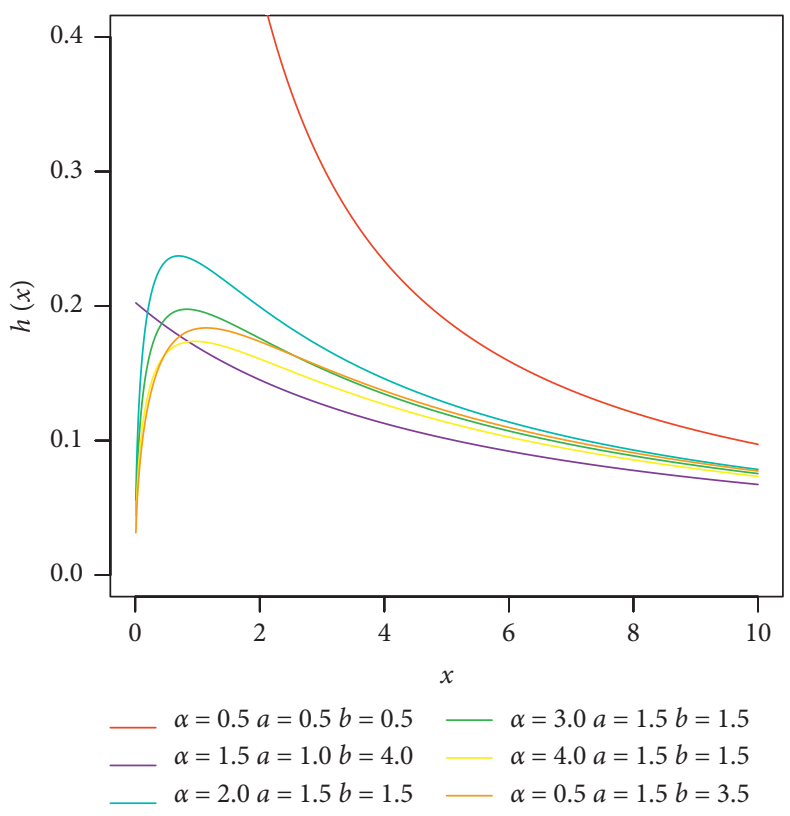

(b)

Figure 1: Plots of the pdf and hrf of the APTIL distribution for different values of parameters.

$$
\begin{aligned}
f(x ; a, b, \alpha) & =\frac{\log (\alpha)}{\alpha-1} \frac{a b}{x^{2}}\left(1+\frac{b}{x}\right)^{-a-1} \alpha^{(1+(b / x))^{-a}} \\
& =\frac{\log (\alpha)}{\alpha-1} \frac{a b}{x^{2}}\left(1+\frac{b}{x}\right)^{-a-1} e^{(1+(b / x))^{-a} \ln a} \\
& =\frac{\log (\alpha)}{\alpha-1} \frac{a b}{x^{2}}\left(1+\frac{b}{x}\right)^{-a-1} \sum_{i=0}^{\infty} \frac{\left\{(1+(b / x))^{-a} \ln a\right\}^{i}}{i !} \\
& =\sum_{i=0}^{\infty} \frac{(\log (\alpha))^{i+1}}{i !(\alpha-1)} \frac{a b}{x^{2}}\left(1+\frac{b}{x}\right)^{-a(i+1)-1},
\end{aligned}
$$$$
f(x ; a, b, \alpha)=\sum_{i=0}^{\infty} w_{i} g_{a(i+1), b}(x), \quad w_{i}=\sum_{i=0}^{\infty} \frac{(\log (\alpha))^{i+1}}{i !(\alpha-1)},
$$

where $g_{a(i+1), b}(x)$ denotes the pdf of IL distribution given in equation (1) with parameters $a(i+1)$ and $b$.

2.2. Quantile Function and Median. The generation from APTIL distribution is obtained by inverting equation (6):

$$
x=b\left[\left\{\frac{\ln \{(\alpha-1) u+1\}}{\ln \alpha}\right\}^{-1 / a}-1\right]^{-1}, \quad \alpha \neq 1, \alpha>0 .
$$

If $q$ uniform $(0,1)$, then $X \operatorname{APTIL}(\alpha, a, b)$, the $q$ th quantile function of $\operatorname{APTIL}(\alpha, a, b)$ is given by

$$
x_{q}=b\left[\left\{\frac{\ln \{(\alpha-1) q+1\}}{\ln \alpha}\right\}^{-1 / a}-1\right]^{-1}
$$

and the median can be obtained as

$$
x_{0.5}=b\left[\left\{\frac{\ln ((\alpha+1) / 2)}{\ln \alpha}\right\}^{-1 / a}-1\right]^{-1},
$$

and when $\alpha=1$, the median of the APTIL distribution is equal to the median of the IL distribution. The analysis of shape of distribution can be performed by study of skewness and kurtosis. Using Bowley's coefficient of skewness as follows:

$$
S_{k}=\frac{x_{0.75}-2 x_{0.5}+x_{0.25}}{x_{0.75}-x_{0.5}}
$$

Moor's coefficient of kurtosis is given as

$$
K=\frac{x_{0.875}-x_{0.625}-x_{0.375}+x_{0.125}}{x_{0.75}-x_{0.5}},
$$

where $x_{(.)}$is the quantile function.

\section{Properties of APTIL Distribution}

This section deals with some statistical properties of APTIL distribution.

3.1. Mode. The mode of APTIL distribution is derived by $f^{\prime}(x)=0$ : 


$$
f^{\prime}(x)= \begin{cases}\frac{a b((b+x) / x)^{-2 a} \alpha^{((b+x) / x)^{-a}} \log (\alpha)\left(((a-1) b-2 x)((b+x) / x)^{a}+a b \log (\alpha)\right)}{x^{2}(b+x)^{2}(\alpha-1)}, & \text { if } \alpha \neq 1, \\ \frac{a b((a-1) b-2 x)((b+x) / x)^{-a}}{x^{2}(b+x)^{2}}, & \text { if } \alpha=1 .\end{cases}
$$

The mode of APTIL distribution cannot be expressed in the closed form. Computer software, e.g., Mathematica or $R$, can be used to compute the mode of APTIL distribution for specific values of parameters.

For $\alpha=1$, the mode of IL distribution can be easily calculated from the following equation:

$$
\frac{a b((a-1) b-2 x)((b+x) / x)^{-a}}{x^{2}(b+x)^{2}}=0 .
$$

3.2. Asymptotic Behavior. The behavior of APTIL distribution is investigated here as $x \longrightarrow 0$ and $x \longrightarrow \infty$ :

$$
\begin{aligned}
\lim _{x \rightarrow 0} f(x) & =\lim _{x \longrightarrow 0} \frac{\log (\alpha)}{\alpha-1} \frac{a b}{x^{2}}\left(1+\left(\frac{b}{x}\right)\right)^{-a-1} \alpha^{(1+(b / x))^{-a}} \\
& =\left\{\begin{array}{ll}
\infty & \text { if } a<1 \\
0 & \text { if } a>1
\end{array},\right. \\
\lim _{x \rightarrow \infty} f(x)= & \lim _{x \longrightarrow \infty} \frac{\log (\alpha)}{\alpha-1} \frac{a b}{x^{2}}\left(1+\frac{b}{x}\right)^{-a-1} \\
& \alpha^{(1+(b / x))^{-a}}=0 .
\end{aligned}
$$

Lemma 1. It can be shown that, for $A P T I L, \lim _{x \rightarrow 0} F(x)=0$ and $\lim _{x \longrightarrow \infty} F(x)=1$

Proof.

$$
\begin{aligned}
& \lim _{x \rightarrow 0} F(x)=\lim _{x \longrightarrow 0} \frac{\alpha^{(1+(b / x))^{-a}}-1}{\alpha-1}=\frac{\alpha^{(\infty)^{-a}}-1}{\alpha-1}=\frac{1-1}{\alpha-1}=0, \\
& \lim _{x \rightarrow \infty} F(x)=\lim _{x \rightarrow \infty} \frac{\alpha^{(1+(b / x))^{-a}}-1}{\alpha-1}=\frac{\alpha^{(1+0)^{-a}}-1}{\alpha-1}=1 .
\end{aligned}
$$

\subsection{Moments}

Theorem 1. Let $X$ be a rv from APTIL distribution, then its $r^{\text {th }}$ moment is

$$
\mu_{r}^{\prime}=a b^{r+1} \sum_{i=0}^{\infty} \frac{(\log (\alpha))^{i+1}}{(\alpha-1) i !} \frac{\Gamma(r+a(i+1)) \Gamma(1-r)}{\Gamma(a(i+1)+1)} .
$$

Proof. Let $X$ be an r.v. with pdf given in equation (5). For any real number $a>0, b>0, \alpha>0$, and $r \geq 0$, the $r^{\text {th }}$ moments of APTIL distribution are obtained as

$$
\mu_{r}^{\prime}=\int_{0}^{\infty} x^{r} f(x ; \alpha, a, b) \mathrm{d} x .
$$

Using expression from equation (10), we get

$$
\begin{aligned}
& \mu_{r}^{\prime}=\int_{0}^{\infty} x^{r} \sum_{i=0}^{\infty} w_{i} g_{a(i+1), b}(x) \mathrm{d} x, \\
& \mu_{r}^{\prime}=\sum_{i=0}^{\infty} w_{i} \frac{a b^{r+1} \Gamma(r+a(i+1)) \Gamma(1-r)}{\Gamma(a(i+1)+1)}, \quad r=1,2,3, \ldots,
\end{aligned}
$$

where $\Gamma(0)=-\gamma, \Gamma(-r)=\left((-1)^{r} / r !\right) \varphi(r)-\left((-1)^{r} / r !\right) \gamma$ for $r=1,2, \ldots, \gamma$ denotes Euler's constant and $\varphi(r)=\sum_{k=1}^{r} 1 / k$ [18]. The mean of $X$ can be obtained using equation (20) by putting $r=1$ :

$$
\mu=\mu_{1}^{\prime}=E(X)=a b^{2} \sum_{i=0}^{\infty} \frac{(\log (\alpha))^{i+1}}{(\alpha-1) i !} \frac{\Gamma(1+a(i+1)) \gamma}{\Gamma(a(i+1)+1)},
$$

where $\gamma$ denotes Euler's constant. The $r^{\text {th }}$ central moment $\mu_{r}$ of $X$ is derived as

$$
\mu_{r}=E\left(X-\mu_{1}^{\prime}\right)^{r}=\sum_{k=0}^{r}\left(\begin{array}{l}
r \\
k
\end{array}\right)(-1)^{r-k} \mu_{r-k}^{\prime}\left(\mu_{1}^{\prime}\right)^{k} .
$$

The variance of APTIL distribution is easily obtained as $\sigma^{2}=\mu_{2}^{\prime}-\left(\mu_{1}^{\prime}\right)^{2}=\frac{a b^{3}}{\alpha-1} \sum_{i=0}^{\infty} \frac{(\log (\alpha))^{i+1}}{i !} \frac{\Gamma(2+a(i+1)) \Gamma(1-r)}{\Gamma(a(i+1)+1)}-\left(\mu_{1}^{\prime}\right)^{2}$.

Lemma 2. Let $X$ be a r. v. with $p d f$ (equation (5)). For any real number $a>0, b>0, \alpha>0, r \geq 0$ and $\delta \geq 0$, the integral,

$$
\tau(a, b, \alpha, r, \delta)=\int_{0}^{\infty} x^{r-2}\left(1+\frac{b}{x}\right)^{-a-1} \alpha^{(1+(b / x))^{-a}} e^{\delta x} \mathrm{~d} x,
$$


is calculated as

$$
\begin{aligned}
\tau(a, b, \alpha, r, \delta)= & \sum_{i=0}^{\infty} \sum_{j=0}^{\infty} \frac{(\delta)^{j}}{j !} \frac{(\log (\alpha))^{i}}{i !}(b)^{r+j-1} \\
& \cdot \frac{\Gamma(r+a(i+1)+j) \Gamma(1-r-j)}{\Gamma(a(i+1)+1)} .
\end{aligned}
$$

Proof of Lemma 2 is given in Appendix.

3.4. Moment Generating Function. The m.g.f. of $X$ is obtained as

$$
M_{x}(t)=\frac{a b}{\alpha-1} \sum_{i=0}^{\infty} \sum_{j=0}^{\infty} \frac{(b t)^{j}}{j !} \frac{(\log (\alpha))^{i+1}}{i !} \frac{\Gamma(a(i+1)+j) \Gamma(1-j)}{\Gamma(a(i+1)+1)} .
$$

Proof. The moment generating function can be derived by

$$
\begin{aligned}
& M_{X}(t)=E\left(e^{t X}\right)=\int_{0}^{\infty} e^{t x} f(x ; a, b, \alpha) \\
& M_{x}(t)=\frac{a b \log (\alpha)}{\alpha-1} \int_{0}^{\infty} e^{t x} x^{-2}\left(1+\frac{b}{x}\right)^{-a-1} \alpha^{(1+(b / x))^{-a}} \mathrm{~d} x
\end{aligned}
$$

Using Lemma 2, the moments, moment generating function, characteristic generating function, and raw moment can be easily obtained by

$$
\begin{aligned}
& M_{X}(t)=\frac{a b \log (\alpha)}{\alpha-1} \tau(a, b, \alpha, 0, t), \\
& \Phi_{X}(t)=\frac{a b \log (\alpha)}{\alpha-1} \tau(a, b, \alpha, 0, i t), \\
& E\left(X^{r}\right)=\frac{a b \log (\alpha)}{\alpha-1} \tau(a, b, \alpha, r, 0) .
\end{aligned}
$$

3.5. Order Statistics. Let $X_{1}, X_{2}, \ldots, X_{n}$ be r.sample from the $\operatorname{APTIL}(\alpha a, b)$ distribution with order statistics $X_{(1)}, X_{(2)}, \ldots, X_{(n)}$. The pdf of $\operatorname{rv} X_{(r)}(r=1,2, \ldots, n)$ is obtained as

$$
\begin{aligned}
f_{X_{(r)}}(x)= & \frac{n !}{(r-1) !(n-r) !} F^{r-1}(x) f(x)\left(1-F^{r-1}(x)\right)^{n-r} \\
f_{X_{(r)}}(x)= & \frac{n ! a b \log (\alpha)}{(r-1) !(n-r) ! x^{2}(\alpha-1)}(1+(b / x))^{-1-a} \\
& \alpha^{(1+(b / x))^{-a}}\left(\frac{\alpha^{(1+(b / x))^{-a}}-1}{\alpha-1}\right)^{r-1}\left(1-\frac{\alpha^{(1+(b / x))^{-a}}-1}{\alpha-1}\right)^{n-r} .
\end{aligned}
$$

The pdf of $X_{(r)}$ can be expressed as

$$
\begin{aligned}
f_{X_{(r)}}(x)= & \frac{n ! a b \log (\alpha)}{(r-1) !(n-r) ! x^{2}(\alpha-1)}\left(\xi^{1-(1 / a)} \alpha^{\xi}\right) \\
& \cdot\left(\frac{\alpha-\alpha^{\xi}}{\alpha-1}\right)^{n-r}\left(\frac{\alpha^{\xi}-1}{\alpha-1}\right)^{r-1},
\end{aligned}
$$

where $(1+(b / x))^{-a}=\xi$. Particularly, pdf of the first and $n^{\text {th }}$ order statistics can be easily derived from equation (32) as

$$
\begin{aligned}
& f_{X_{(1)}}(x)=\frac{n a b \log (\alpha)}{x^{2}(\alpha-1)}\left(\xi^{1-(1 / a)} \alpha^{\xi}\right)\left(\frac{\alpha-\alpha^{\xi}}{\alpha-1}\right)^{n-1}, \\
& f_{X_{(n)}}(x)=\frac{n a b \log (\alpha)}{x^{2}(\alpha-1)}\left(\xi^{1-(1 / a)} \alpha^{\xi}\right)\left(\frac{\alpha^{\xi}-1}{\alpha-1}\right)^{n-1},
\end{aligned}
$$

respectively.

3.6. Stress-Strength Model. Let $X_{1}$ and $X_{2}$ be two independent random variables with $\operatorname{APTIL}\left(\alpha_{1}, a_{1}, b_{1}\right)$ and $\operatorname{APTIL}\left(\alpha_{2}, a_{2}, b_{2}\right)$ distributions, respectively. If $X_{1}$ represents "stress" and $X_{2}$ represents "strength," the reliability is defined

$R=P\left(X_{2}>X_{1}\right)=\int_{0}^{\infty} f_{2}\left(x ; \alpha_{2}, a_{2}, b_{2}\right) F_{1}\left(x ; \alpha_{1}, a_{1}, b_{1}\right) \mathrm{d} x$.

Then, we can write

$$
\begin{aligned}
& \mathbf{R}=\frac{a_{2} b_{2} \log \left(\alpha_{2}\right)}{\left(\alpha_{1}-1\right)\left(\alpha_{2}-1\right)} \int_{0}^{\infty}\left(x^{-2}\left(1+\frac{b_{2}}{x}\right)^{-1-a_{2}} \alpha_{2}^{\left(1+\left(b_{2} / x\right)\right)^{-a_{2}}}\right. \\
& \left.\cdot\left(\alpha_{1}^{\left(1+\left(b_{1} / x\right)\right)^{-a 1}}-1\right)\right) \mathrm{d} x \\
& =\frac{a_{2} b_{2} \log \left(\alpha_{2}\right)}{\left(\alpha_{1}-1\right)\left(\alpha_{2}-1\right)} \int_{0}^{\infty}\left(x^{-2}\left(1+\frac{b_{2}}{x}\right)^{-1-a_{2}} \alpha_{2}^{\left(1+\left(b_{2} / x\right)\right)^{-a_{2}}}\right. \\
& \left.\cdot \alpha_{1}^{\left(1+\left(b_{1} / x\right)\right)^{-a_{1}}}-x^{-2}\left(1+\frac{b_{2}}{x}\right)^{-1-a_{2}} \alpha_{2}^{\left(1+\left(b_{2} / x\right)\right)^{-a_{2}}}\right) \mathrm{d} x \text {. }
\end{aligned}
$$

Using Lemma 2 from equation (27), we have 


$$
\begin{aligned}
= & \frac{a_{2} b_{2} \log \left(\alpha_{2}\right)}{\left(\alpha_{1}-1\right)\left(\alpha_{2}-1\right)} \int_{0}^{\infty} \alpha_{1}^{\left(1+\left(b_{1} / x\right)\right)^{-a_{1}}}\left(x^{-2}\left(1+\frac{b_{2}}{x}\right)^{-1-a_{2}} \alpha_{2}^{\left(1+\left(b_{2} / x\right)\right)^{-a_{2}}}\right) \mathrm{d} x-\frac{a_{2} b_{2} \log \left(\alpha_{2}\right)}{\left(\alpha_{1}-1\right)\left(\alpha_{2}-1\right)} \tau\left(a_{2}, b_{2}, \alpha_{2}, 0,0\right) \\
= & \frac{1}{\left(\alpha_{1}-1\right)}\left\{\frac{a_{2} b_{2} \log \left(\alpha_{2}\right)}{\left(\alpha_{2}-1\right)} \int_{0}^{\infty} \alpha_{1}^{\left(1+\left(b_{1} / x\right)\right)^{-a_{1}}}\left(x^{-2}\left(1+\frac{b_{2}}{x}\right)^{-1-a_{2}} \alpha_{2}^{\left(1+\left(b_{2} / x\right)\right)^{-a_{2}}} \alpha_{1}^{\left.\left(1+\left(b_{1} / x\right)\right)^{-a 1}\right)} \mathrm{d} x\right\}\right. \\
& -\frac{a_{2} b_{2} \log \left(\alpha_{2}\right)}{\left(\alpha_{1}-1\right)\left(\alpha_{2}-1\right)} \tau\left(a_{2}, b_{2}, \alpha_{2}, 0,0\right) \\
\mathbf{R}= & \frac{1}{\left(\alpha_{1}-1\right)} E\left[\alpha_{1}^{\left(1+\left(b_{1} / x\right)\right)^{-a_{1}}}\right]-\frac{a_{2} b_{2} \log \left(\alpha_{2}\right)}{\left(\alpha_{1}-1\right)\left(\alpha_{2}-1\right)} \tau\left(a_{2}, b_{2}, \alpha_{2}, 0,0\right) .
\end{aligned}
$$

The effect of parameters $a, b$, and $\alpha$ on mean, variance, skewness, and kurtosis is displayed in Figures 2 and 3, respectively.

\section{Remark 1}

(i) The mean and variance of APTIL distribution increase as " $a$ " or " $b$ " increase for fixed value of $\alpha$

(ii) For increasing $\alpha$, the mean of distribution decreases as " $b$ " decreases for higher value of " $a$ " and increases for lower value of " $a$ "

(iii) For increasing $\alpha$, the variance of distribution increases as " $b$ " increases for lower value of " $a$ " (iv) The skewness and kurtosis of APTIL distribution decrease as " $a$ " increases or " $b$ " decreases for fixed value of $\alpha$

3.7. Characterization Based on Hazard Function. In this section, characterizations of APTIL distribution based on the hazard function are presented. It is known that hazard function $h(x)$ satisfies the following differential equation:

$$
\frac{f^{\prime}(x)}{f(x)}=\frac{h^{\prime}(x)}{h(x)}-h(x)
$$

Theorem 2. Let $X:(0, \infty)$ be a continuous $r . v$. with $p d f(5)$ iff its $h r f h(x)$ satisfies the differential equation:

$$
h \prime(x)-(a+1) x^{-2}\left(1+\frac{b}{x}\right)^{-1} h(x)=\frac{a b((b+x) / x)^{-2 a} \alpha^{((b+x) / x)^{-a}} \log (\alpha)\left\{2(b+x)((b+x) / x)^{a}\left(-\alpha+\alpha^{((b+x) / x)^{-a}}\right)+a b \alpha \ln (\alpha)\right\}}{x^{2}(b+x)^{2}\left(\alpha-\alpha^{((b+x) / x)^{-a}}\right)^{2}}
$$

under the boundary conditions $h(0) \geq 0$.

Proof. If rv $X$ has the hrf given in (8), then

$$
\begin{aligned}
& h^{\prime}(x)=\frac{a b((b+x) / x)^{-2 a} \alpha^{((b+x) / x)^{-a}} \log (\alpha)\left[\{ ( a - 1 ) b - 2 x \} ( ( b + x ) / x ) ^ { a } \left(-\alpha+\alpha^{\left.\left.((b+x) / x)^{-a}\right)+a b \alpha \ln (\alpha)\right]}\right.\right.}{x^{2}(b+x)^{2}\left(\alpha-\alpha^{((b+x) / x)^{-a}}\right)^{2}}, \\
& h^{\prime}(x)-(a+1) x^{-2}\left(1+\frac{b}{x}\right)^{-1} h(x)=-\frac{a(1+a) b^{2}(1+(b / x))^{-a-2} \alpha^{(1+(b / x))^{-a}} \log (\alpha)}{x^{4}(\alpha-1)\left(1-\left(\alpha^{\left.\left.(1+(b / x))^{-a}-1 / \alpha-1\right)\right)}\right.\right.} \\
& +\left\{\frac{a b((b+x) / x)^{-2 a} \alpha^{((b+x) / x)^{-a}} \log (\alpha)\left[-\{(a-1) b-2 x\}((b+x) / x)^{a}\left(-\alpha+\alpha^{((b+x) / x)^{-a}}\right)+a b \alpha \log (\alpha)\right]}{x^{2}(b+x)^{2}\left(\alpha-\alpha^{((b+x) / x)^{-a}}\right)^{2}}\right\} .
\end{aligned}
$$


Now the result follows.

Conversely if equation (38) holds, then

$$
\begin{aligned}
\frac{\mathrm{d}}{\mathrm{d} x}\left[(1+(b / x))^{a+1} h(x)\right] & =\frac{\mathrm{d}}{\mathrm{d} x}\left[\frac{a b(1+(b / x))^{1+a}((b+x) / x)^{-1-a} \alpha^{((b+x) / x)^{-a}} \log (\alpha)}{x^{2}\left\{\alpha-\alpha^{((b+x) / x)^{-a}}\right\}}\right], \\
h(x) & =\frac{a b((b+x) / x)^{-1-a} \alpha^{((b+x) / x)^{-a}} \log (\alpha)}{x^{2}\left\{\alpha-\alpha^{((b+x) / x)^{-a}}\right\}}+C
\end{aligned}
$$

which implies $C=0$.

\section{Estimation of Parameters}

The parameters of the APTIL distribution are estimated using various methods including maximum likelihood (ML), least squares (LS), weighted least squares (WLS), percentile (PC), Cramer-von Mises (CV), maximum product of spacing (MPS), Anderson-Darling (AD), and right-tail Anderson-Darling (RTAD) methods of estimation.

4.1. ML Estimation. Let $X_{1}, \ldots, X_{n}$ have the observed values from APTIL distribution. The MLEs of the proposed model parameters $\alpha, a$, and $b$ are derived using the $\log$-likelihood function say $\ell$ which is given by

$$
\begin{aligned}
\ell= & n \ln (\log (\alpha))-n \ln (\alpha-1)+n \ln (a)+n \ln (b) \\
& -2 \sum_{i=1}^{n} \log \left(x_{i}\right)-(a+1) \sum_{i=1}^{n} \ln \left(1+\frac{b}{x_{i}}\right)+\ln (\alpha) \sum_{i=1}^{n}\left(1+\frac{b}{x_{i}}\right)^{-a} .
\end{aligned}
$$

The ML equations of the APTIL distribution are given by

$$
\begin{aligned}
& \frac{\partial \ell}{\partial \alpha}=\frac{n}{\alpha \log (\alpha)}-\frac{n}{\alpha-1}+\frac{1}{\alpha} \sum_{i=1}^{n}\left(1+\frac{b}{x_{i}}\right)^{-a}, \\
& \frac{\partial \ell}{\partial a}=\frac{n}{a}-\sum_{i=1}^{n} \ln \left(1+\frac{b}{x_{i}}\right)-\ln (\alpha) \sum_{i=1}^{n}\left(1+\frac{b}{x_{i}}\right)^{-a} \ln \left(1+\frac{b}{x_{i}}\right), \\
& \frac{\partial \ell}{\partial b}=\frac{n}{b}-(a+1) \sum_{i=1}^{n} \frac{1}{\left(x_{i}+b\right)}+a \ln (\alpha)\left(1+\frac{b}{x_{i}}\right)^{-a-1} \frac{1}{\left(x_{i}\right)} .
\end{aligned}
$$

Equating $\partial \ell / \partial \alpha, \partial \ell / \partial a$, and $\partial \ell / \partial b$ with zeros and solving simultaneously, we obtain the ML estimators of $\alpha, a$, and $b$.

4.2. Ordinary and Weighted LS Estimators. Suppose $X_{1}, X_{2}, \ldots, X_{n}$ is a random sample from APTIL distribution with corresponding ordered sample of $X_{(1)}, X_{(2)}, \ldots, X_{(n)}$. The mean and variance of APTIL are independent of unknown parameter and are as follows:

$$
\begin{aligned}
E\left(F\left(X_{(i)}\right)\right) & =\frac{i}{n+1}, \\
\operatorname{var}\left(F\left(X_{(i)}\right)\right) & =\frac{i(n-i+1)}{(n+1)^{2}(n+2)},
\end{aligned}
$$

where $F\left(X_{(i)}\right)$ is the cdf of APTIL distribution with $X_{(i)}$ being the $i^{\text {th }}$ order statistic. Then, LS estimators ([19]) are obtained by minimizing the SSE:

$$
\sum_{i=1}^{n}\left[F_{(i)}(x)-\frac{i}{n+1}\right]^{2},
$$

with respect to $\alpha, a$, and $b$. So, the LS estimators (LSEs) of the parameters $\alpha, a$, and $b$ of the APTIL are obtained by minimizing the following:

$$
\sum_{i=1}^{n}\left[\frac{\alpha^{\left(1+\left(b / x_{(i)}\right)\right)^{-a}-1}}{\alpha-1}-\frac{i}{n+1}\right]^{2},
$$

with respect to $\alpha, a$, and $b$.

The WLS estimators [19] of $\alpha, a$, and $b$ can be obtained by minimizing the following expression:

$$
\sum_{i=1}^{n} \frac{(n+1)^{2}(n+2)}{i(n-i+1)}\left[\frac{\alpha^{\left(1+\left(b / x_{(i)}\right)\right)^{-a}}-1}{\alpha-1}-\frac{i}{n+1}\right]^{2},
$$

with respect to $\alpha, a$, and $b$.

4.3. PC Estimator (PCE). Let $X_{(1)}<X_{(2)}<\cdots<X_{(n)}$ be order statistics. Using on PC method of estimation $([20,21])$, the estimators of $\alpha, a$, and $b$ are derived by minimizing the following:

$$
\sum_{i=1}^{n}\left[\ln \left(\frac{i}{n+1}\right)-\ln \left(\frac{\alpha^{\left(1+\left(b / x_{(i)}\right)\right)^{-a}}-1}{\alpha-1}\right)\right]^{2},
$$

with respect to $\alpha, a$, and $b$.

4.4. The Cramer-von Mises Minimum Distance Estimators. The CV method is based on the difference between the estimated cdf and the empirical cdf $([22,23])$. The CV estimators are obtained by minimizing

$$
\mathrm{CV}=\frac{1}{12 n}+\sum_{i=1}^{n}\left[\frac{\alpha^{\left(1+\left(b / x_{(i)}\right)\right)^{-a}-1}}{\alpha-1}-\frac{2 i-1}{2 n}\right]^{2} .
$$




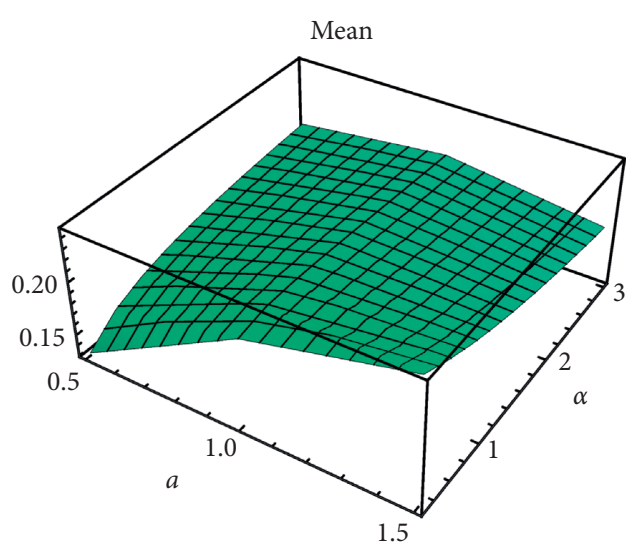

(a)

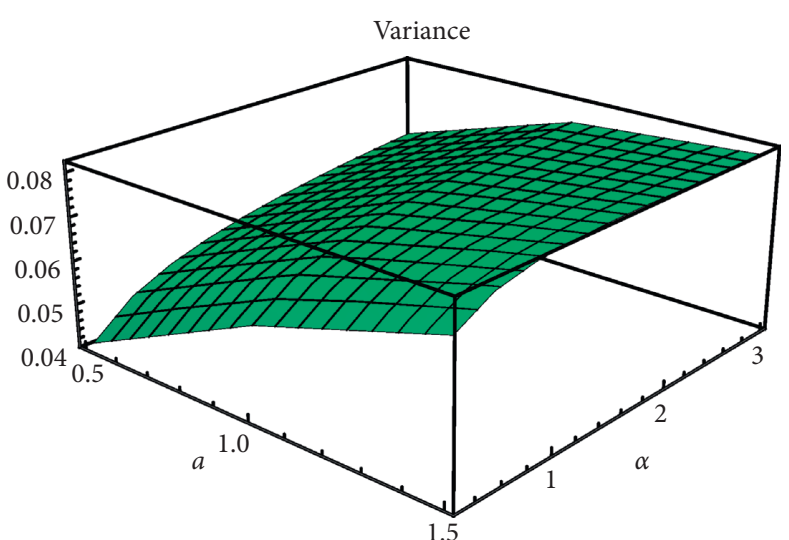

(b)

Figure 2: Plots of the mean and variance for the APTIL distribution.

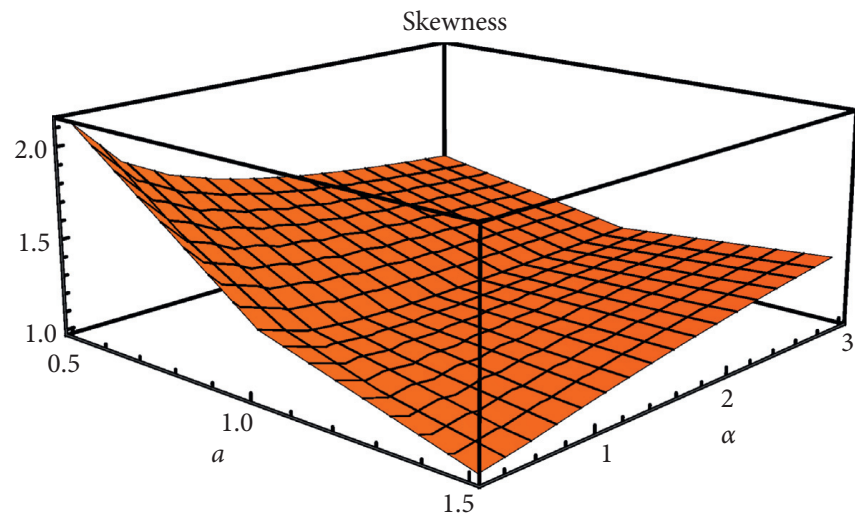

(a)

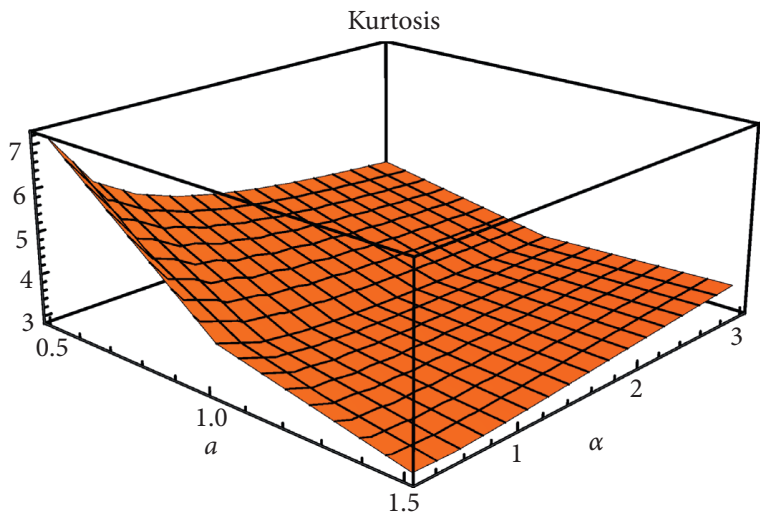

(b)

FIgURE 3: Plots of the skewness and kurtosis for the APTIL distribution.

Macdonald [24] stated about the CV method that it depends on minimum distance estimators providing empirical evidence that the bias of the estimator is smaller than the other minimum distance estimators.

4.5. Maximum Product of Spacing Estimators. The MPS method is a powerful alternative to the ML method for estimating the population parameters of continuous distributions ([25]). Let

$$
\begin{array}{r}
D_{i}(\alpha, a, b)=F\left(x_{(i)} \mid \alpha, a, b\right)-F\left(x_{(i-1)} \mid \alpha, a, b\right), \\
i=1,2, \ldots, n+1,
\end{array}
$$

be the uniform spacings of a random sample from the APTL distribution, where

$$
\begin{aligned}
F\left(x_{(0)} \mid \alpha, a, b\right) & =0, \\
F\left(x_{(n+1)} \mid \alpha, a, b\right) & =1, \\
\sum_{i=1}^{n+1} D_{i}(\alpha, a, b) & =1 .
\end{aligned}
$$

The MPS estimator is obtained by maximizing the geometric mean (GM) of the spacings:

$$
\operatorname{GM}(\alpha, a, b)=\left\{\prod_{i=1}^{n+1} D_{i}(\alpha, a, b)\right\}^{1 / n+1},
$$

w.r.t. $\alpha, a$, and $b$. The MPS estimator of $\alpha, a$, and $b$ can be obtained by maximizing the logarithm of the GM of sample spacing's equation (51). No closed solution exists, so the numerical method is used to find the estimates.

4.6. Anderson-Darling and Right-Tail Anderson-Darling Estimators. The method of Anderson-Darling estimation was introduced by [26] in the context of statistical tests. By adapting it to the APTIL model, the Anderson-Darling estimates (ADEs) of $\alpha, a$, and $b$ can be obtained by minimizing, with respect to $\alpha, a$, and $b$, the function given by

$$
\begin{aligned}
\mathrm{AD}= & -n-\frac{1}{n} \sum_{i=1}^{n}(2 i-1)\left\{\log \left[F\left(x_{(i)} ; \alpha, a, b\right)\right]\right. \\
& \left.+\log \left[1-F\left(x_{(n+1-i)} ; \alpha, a, b\right)\right]\right\} .
\end{aligned}
$$


TABLE 1: Estimates and MSEs of APTIL distribution for ML, LS, WLS, CV, PC, MPS, AD, and RTAD estimates for set 1.

\begin{tabular}{|c|c|c|c|c|c|c|c|c|c|c|c|c|c|c|c|c|}
\hline \multirow{3}{*}{$n$} & \multicolumn{16}{|c|}{ Set 1: $\alpha=0.5, b=0.5, a=2$} \\
\hline & \multicolumn{2}{|c|}{ MLEs } & \multicolumn{2}{|c|}{ LSEs } & \multicolumn{2}{|c|}{ WLSES } & \multicolumn{2}{|c|}{ CVEs } & \multicolumn{2}{|c|}{ PCEs } & \multicolumn{2}{|c|}{ MPSEs } & \multicolumn{2}{|c|}{ ADEs } & \multicolumn{2}{|c|}{ RTADEs } \\
\hline & Est. & MSEs & Est. & MSEs & Est. & MSEs & Est. & MSEs & Est. & MSEs & Est. & MSEs & Est. & MSEs & Est. & MSEs \\
\hline \multirow{3}{*}{50} & 0.451 & 0.16 & 0.491 & 0.181 & 0.542 & 0.276 & 0.468 & 0.246 & 0.587 & 0.496 & 0.709 & 0.489 & 0.432 & 0.089 & 0.429 & 0.184 \\
\hline & 0.697 & 0.202 & 0.910 & 0.261 & 0.827 & 0.197 & 0.848 & 0.199 & 0.836 & 0.300 & 0.882 & 0.600 & 0.806 & 0.173 & 1.024 & 0.399 \\
\hline & 2.151 & 0.499 & 1.561 & 0.346 & 1.695 & 0.345 & 1.693 & 0.243 & 1.789 & 0.536 & 1.998 & 1.069 & 1.725 & 0.223 & 1.530 & 0.368 \\
\hline \multirow{3}{*}{100} & 0.401 & 0.084 & 0.394 & 0.123 & 0.447 & 0.197 & 0.401 & 0.120 & 0.386 & 0.065 & 0.577 & 0.232 & 0.395 & 0.066 & 0.411 & 0.139 \\
\hline & 0.642 & 0.076 & 0.825 & 0.147 & 0.772 & 0.138 & 0.813 & 0.154 & 0.845 & 0.309 & 0.767 & 0.372 & 0.711 & 0.083 & 0.905 & 0.235 \\
\hline & 1.983 & 0.135 & 1.684 & 0.235 & 1.803 & 0.274 & 1.737 & 0.278 & 1.798 & 0.298 & 1.93 & 0.318 & 1.828 & 0.135 & 1.628 & 0.317 \\
\hline \multirow{3}{*}{200} & 0.394 & 0.068 & 0.374 & 0.104 & 0.409 & 0.136 & 0.352 & 0.099 & 0.342 & 0.050 & 0.496 & 0.121 & 0.383 & 0.044 & 0.362 & 0.106 \\
\hline & 0.68 & 0.069 & 0.798 & 0.122 & 0.735 & 0.094 & 0.797 & 0.117 & 0.773 & 0.192 & 0.687 & 0.168 & 0.671 & 0.055 & 0.857 & 0.157 \\
\hline & 1.861 & 0.082 & 1.683 & 0.154 & 1.785 & 0.137 & 1.706 & 0.146 & 1.797 & 0.142 & 1.902 & 0.155 & 1.833 & 0.076 & 1.607 & 0.191 \\
\hline
\end{tabular}

TABLE 2: Estimates and MSEs of APTIL distribution for ML, LS, WLS, CV, PC, MPS, AD, and RTAD estimates for set 2.

\begin{tabular}{|c|c|c|c|c|c|c|c|c|c|c|c|c|c|c|c|c|}
\hline \multirow{3}{*}{$n$} & \multicolumn{16}{|c|}{ Set 2: $\alpha=0.5, b=0.5, a=3$} \\
\hline & \multicolumn{2}{|c|}{ MLEs } & \multicolumn{2}{|c|}{ LSEs } & \multicolumn{2}{|c|}{ WLSEs } & \multicolumn{2}{|c|}{ CVEs } & \multicolumn{2}{|c|}{ PCEs } & \multicolumn{2}{|c|}{ MPSEs } & \multicolumn{2}{|c|}{ ADEs } & \multicolumn{2}{|c|}{ RTADEs } \\
\hline & Est. & MSEs & Est. & MSEs & Est. & MSEs & Est. & MSEs & Est. & MSEs & Est. & MSEs & Est. & MSEs & Est. & MSEs \\
\hline \multirow{3}{*}{50} & 0.472 & 0.186 & 0.709 & 1.148 & 0.64 & 1.163 & 0.518 & 0.803 & 0.364 & 0.086 & 0.56 & 0.237 & 0.377 & 0.110 & 0.810 & 1.526 \\
\hline & 0.752 & 0.204 & 1.094 & 0.623 & 1.088 & 0.523 & 1.175 & 0.724 & 1.104 & 0.651 & 0.981 & 0.670 & 1.057 & 0.438 & 1.118 & 0.717 \\
\hline & 2.778 & 0.540 & 2.227 & 1.644 & 2.172 & 1.227 & 2.311 & 1.599 & 2.169 & 1.018 & 2.532 & 1.325 & 2.183 & 0.880 & 2.252 & 1.447 \\
\hline \multirow{3}{*}{100} & 0.473 & 0.159 & 0.525 & 0.569 & 0.52 & 0.970 & 0.591 & 0.900 & 0.311 & 0.076 & 0.482 & 0.187 & 0.313 & 0.082 & 0.721 & 1.006 \\
\hline & 0.738 & 0.182 & 1.113 & 0.612 & 1.035 & 0.406 & 1.136 & 0.622 & 1.068 & 0.549 & 0.971 & 0.554 & 1.046 & 0.415 & 0.995 & 0.508 \\
\hline & 2.728 & 0.354 & 2.303 & 1.481 & 2.291 & 1.161 & 2.236 & 1.508 & 2.211 & 0.855 & 2.434 & 0.944 & 2.148 & 0.816 & 2.411 & 1.425 \\
\hline \multirow{3}{*}{200} & 0.454 & 0.085 & 0.521 & 0.595 & 0.446 & 0.660 & 0.292 & 0.337 & 0.262 & 0.082 & 0.384 & 0.087 & 0.261 & 0.074 & 0.498 & 0.463 \\
\hline & 0.664 & 0.100 & 1.078 & 0.572 & 0.995 & 0.308 & 1.257 & 0.692 & 1.007 & 0.484 & 0.833 & 0.263 & 0.987 & 0.309 & 1.032 & 0.512 \\
\hline & 2.771 & 0.287 & 2.481 & 1.798 & 2.281 & 1.067 & 2.054 & 1.221 & 2.333 & 0.609 & 2.517 & 0.491 & 2.231 & 0.655 & 2.417 & 1.229 \\
\hline
\end{tabular}

TABLE 3: Estimates and MSEs of APTIL distribution for ML, LS, WLS, CV, PC, MPS, AD, and RTAD estimates for set 3.

\begin{tabular}{|c|c|c|c|c|c|c|c|c|c|c|c|c|c|c|c|c|}
\hline \multirow{3}{*}{$n$} & \multicolumn{16}{|c|}{ Set 3: $\alpha=0.3, b=0.5, a=2$} \\
\hline & \multicolumn{2}{|c|}{ MLEs } & \multicolumn{2}{|c|}{ LSEs } & \multicolumn{2}{|c|}{ WLSEs } & \multicolumn{2}{|c|}{ CVEs } & \multicolumn{2}{|c|}{ PCEs } & \multicolumn{2}{|c|}{ MPSEs } & \multicolumn{2}{|c|}{ ADEs } & \multicolumn{2}{|c|}{ RTADEs } \\
\hline & Est. & MSEs & Est. & MSEs & Est. & MSEs & Est. & MSEs & Est. & MSEs & Est. & MSEs & Est. & MSEs & Est. & MSEs \\
\hline \multirow{3}{*}{50} & 0.315 & 0.053 & 0.26 & 0.066 & 0.263 & 0.085 & 0.212 & & 0.401 & 0.604 & 0.413 & 0.234 & 0.282 & 0.052 & 0.234 & 0.056 \\
\hline & 0.509 & 0.032 & 0.805 & 0.128 & 0.854 & 0.19 & 0.895 & 0.205 & 0.798 & 0.255 & 0.849 & 0.565 & 0.71 & 0.126 & 0.948 & 0.285 \\
\hline & 2.243 & 0.356 & 1.628 & & 1.697 & & 1.678 & & & & 1.878 & & 1.838 & & & 0.26 \\
\hline \multirow{3}{*}{100} & 0.325 & 0.029 & 0.221 & 0.025 & 0.234 & 0.035 & 0.181 & 0.033 & 0.303 & 0.06 & 0.387 & 0.171 & 0.27 & 0.039 & 0.199 & 0.033 \\
\hline & 0.504 & 0.033 & 0.789 & 0.113 & 0.771 & 0.111 & 0.838 & & 0.737 & & 0.682 & 0.198 & 0.637 & 0.058 & 0.872 & 0.178 \\
\hline & 2.138 & 0.217 & 1.67 & 0.149 & 1.724 & 0.177 & 1.686 & 0.135 & 1.864 & 0.321 & 1.96 & 0.257 & 1.906 & 0.095 & 1.616 & 0.179 \\
\hline \multirow{3}{*}{200} & 0.336 & 0.016 & 0.228 & 0.026 & 0.218 & 0.026 & 0.179 & & 0.29 & & 0.409 & 0.149 & 0.274 & 0.021 & 0.211 & 0.022 \\
\hline & 0.48 & $8.602^{*}$ & 0.791 & 0.099 & 0.74 & 0.087 & 0.788 & 0.096 & 0.65 & 0.105 & 0.596 & 0.121 & 0.602 & 0.037 & 0.812 & 0.119 \\
\hline & 2.081 & 0.076 & 1.632 & 0.156 & 1.763 & 0.083 & 1.702 & 0.109 & 1.907 & 0.145 & 2.031 & 0.204 & 1.906 & 0.065 & 1.624 & 0.163 \\
\hline
\end{tabular}

TABLE 4: Estimates and MSEs of APTIL distribution for ML, LS, WLS, CV, PC, MPS, AD, and RTAD estimates for set 4.

\begin{tabular}{|c|c|c|c|c|c|c|c|c|c|c|c|c|c|c|c|c|}
\hline \multirow{3}{*}{$n$} & \multicolumn{16}{|c|}{ Set $4: \alpha=0.3, b=0.5, a=3$} \\
\hline & \multicolumn{2}{|c|}{ MLEs } & \multicolumn{2}{|c|}{ LSEs } & \multicolumn{2}{|c|}{ WLSEs } & \multicolumn{2}{|c|}{ CVEs } & \multicolumn{2}{|c|}{ PCEs } & \multicolumn{2}{|c|}{ MPSEs } & \multicolumn{2}{|c|}{ ADEs } & \multicolumn{2}{|c|}{ RTADEs } \\
\hline & Est. & MSES & Est. & MSEs & Est. & MSEs & Est. & MSEs & Est. & MSEs & Est. & MSEs & Est. & MSEs & Est. & MSEs \\
\hline \multirow{3}{*}{50} & 0.306 & 0.093 & 0.544 & 0.711 & 0.561 & 0.916 & 0.339 & 0.366 & 0.266 & 0.079 & 0.497 & 0.468 & 0.259 & 0.575 & 0.380 & 0.260 \\
\hline & 0.753 & 0.166 & 0.968 & 0.511 & 0.951 & 0.410 & 0.913 & 0.435 & 1.032 & 0.539 & 0.865 & 0.513 & 0.965 & 0.819 & 1.047 & 0.589 \\
\hline & 2.684 & 0.410 & 2.440 & 1.512 & 2.425 & 1.552 & 2.668 & 1.441 & 2.309 & 0.850 & 2.695 & 0.972 & 2.270 & 1.689 & 2.391 & 1.282 \\
\hline \multirow{3}{*}{100} & 0.304 & 0.086 & 0.312 & 0.242 & 0.399 & 0.474 & 0.328 & 0.304 & 0.194 & 0.031 & 0.325 & 0.094 & 0.217 & 0.427 & 0.39 & 0.310 \\
\hline & 0.751 & 0.153 & 0.949 & 0.397 & 0.934 & 0.342 & 0.986 & 0.427 & 0.998 & 0.501 & 0.819 & 0.327 & 0.864 & 0.680 & 0.916 & 0.375 \\
\hline & 2.585 & 0.353 & 2.511 & 1.250 & 2.516 & 1.833 & 2.436 & 1.296 & 2.323 & 0.736 & 2.626 & 0.807 & 2.310 & 1.547 & 2.478 & 1.086 \\
\hline \multirow{3}{*}{200} & 0.251 & 0.033 & 0.228 & 0.073 & 0.250 & & 0.398 & 0.311 & 0.164 & 0.029 & 0.326 & 0.076 & 0.197 & 0.227 & 0.397 & 0.221 \\
\hline & 0.69 & 0.081 & 0.990 & 0.394 & 1.026 & 0.399 & 0.881 & 0.380 & 0.885 & 0.276 & 0.696 & 0.168 & 0.872 & 0.482 & 0.780 & 0.288 \\
\hline & 2.667 & 0.238 & 2.412 & 1.160 & 2.388 & 1.406 & 2.766 & 1.290 & 2.500 & 0.441 & 2.813 & 0.477 & 2.332 & 1.489 & 2.781 & 0.908 \\
\hline
\end{tabular}


TABLE 5: Partial and overall ranks of all the methods of estimation for various combination of parameters.

\begin{tabular}{|c|c|c|c|c|c|c|c|c|c|}
\hline Parameters & $n$ & ML & LS & WLS & $\mathrm{CV}$ & PC & MPS & $\mathrm{AD}$ & RTAD \\
\hline \multirow{3}{*}{$(\alpha=0.5, b=0.5, a=2)$} & 50 & 4.5 & 4.5 & 3 & 2 & 7 & 8 & 1 & 6 \\
\hline & 100 & 1.5 & 3 & 5 & 5 & 5 & 8 & 1.5 & 7 \\
\hline & 200 & 2 & 6 & 4.5 & 3 & 4.5 & 7 & 1 & 8 \\
\hline \multirow{3}{*}{$(\alpha=0.5, b=0.5, a=3)$} & 50 & 1 & 6 & 4 & 7 & 3 & 5 & 2 & 8 \\
\hline & 100 & 1 & 6 & 4.5 & 8 & 3 & 4.5 & 2 & 6 \\
\hline & 200 & 1 & 8 & 5 & 6.5 & 4 & 2 & 3 & 6.5 \\
\hline \multirow{3}{*}{$(\alpha=0.3, b=0.5, a=2)$} & 50 & 2.5 & 4 & 5 & 2.5 & 7 & 8 & 1 & 6 \\
\hline & 100 & 3 & 1.5 & 5 & 4 & 8 & 7 & 1.5 & 6 \\
\hline & 200 & 1 & 5 & 3 & 4 & 7 & 8 & 2 & 6 \\
\hline \multirow{3}{*}{$(\alpha=0.3, b=0.5, a=3)$} & 50 & 1 & 6.5 & 6.5 & 3 & 2 & 4 & 8 & 5 \\
\hline & 100 & 1 & 4.5 & 7 & 6 & 3 & 2 & 8 & 4.5 \\
\hline & 200 & 1 & 4.5 & 6.5 & 6.5 & 2 & 3 & 8 & 4.5 \\
\hline$\sum$ Ranks & & 20.5 & 59.5 & 59 & 57.5 & 55.5 & 66.5 & 39 & 73.5 \\
\hline Overall rank & & 1 & 6 & 5 & 4 & 3 & 7 & 2 & 8 \\
\hline
\end{tabular}

TABLE 6: Estimated values for the data sets.

\begin{tabular}{|c|c|c|c|}
\hline \multirow{2}{*}{ Model } & \multicolumn{3}{|c|}{ Estimates } \\
\hline & \multicolumn{3}{|c|}{ Data I } \\
\hline $\operatorname{APTIL}(\alpha, a, b)$ & 0.000439 & 709.021 & 0.146377 \\
\hline $\operatorname{APW}(\alpha, \beta, \lambda)$ & 11.22380 & 1.28552 & 0.010140 \\
\hline $\operatorname{APIE}(\alpha, \lambda)$ & $5.10175 * 10^{10}$ & 1.62081 & \\
\hline $\operatorname{APP}(\alpha, \beta)$ & 198.1017 & 0.59646 & \\
\hline $\operatorname{MOLBE}(\alpha, \beta)$ & 2.270760 & 18.7701 & \\
\hline $\operatorname{IL}(a, b)$ & 902.7830 & 0.04409 & \\
\hline \multicolumn{4}{|c|}{ Data II } \\
\hline $\operatorname{APTIL}(\alpha, a, b)$ & 0.000327 & 3.28047 & 22.8655 \\
\hline $\operatorname{APIW}(\alpha, \beta, \lambda)$ & 24.49500 & 1.72561 & 37.9214 \\
\hline $\operatorname{APIE}(\alpha, \lambda)$ & 0.041375 & 29.3658 & \\
\hline $\operatorname{APP}(\alpha, \beta)$ & 257.0863 & 0.75805 & \\
\hline $\operatorname{MOLBE}(\alpha, \beta)$ & 0.823351 & 13.9648 & \\
\hline $\operatorname{IL}(a, b)$ & 2431.360 & 0.00646 & \\
\hline \multicolumn{4}{|c|}{ Data III } \\
\hline $\operatorname{APTIL}(\alpha, a, b)$ & $5.72005 * 10^{-8}$ & 0.90389 & 1136.77 \\
\hline $\operatorname{APIW}(\alpha, \beta, \lambda)$ & 84.1137 & 0.91645 & 3.32642 \\
\hline $\operatorname{APIE}(\alpha, \lambda)$ & 101.484 & 3.93250 & \\
\hline $\operatorname{APP}(\alpha, \beta)$ & 964.635 & 0.72033 & \\
\hline $\operatorname{MOLBE}(\alpha, \beta)$ & 0.06136 & 74.9680 & \\
\hline $\operatorname{IL}(a, b)$ & 1.87063 & 12.6355 & \\
\hline
\end{tabular}

Similarly, the right-tail Anderson-Darling estimates (RTADEs) of $\alpha, a$, and $b$ can be obtained by minimizing, with respect to $\alpha, a$, and $b$, the function given by

$$
\begin{aligned}
\operatorname{RTAD}= & \frac{n}{2}-2 \sum_{i=1}^{n}\left\{\log \left[F\left(x_{(i)} ; \alpha, a, b\right)\right]\right\} \\
& -\frac{1}{n} \sum_{i=1}^{n}(2 i-1)\left\{\log \left[1-F\left(x_{(n+1-i)} ; \alpha, a, b\right)\right]\right\} .
\end{aligned}
$$

\section{Simulation Study}

Here, we come up with a numerical study to compare the behavior of different estimates. We generate 1000 random samples of size $n=50,100$, and 200 from the APTIL distribution. Four sets of the parameters are assigned as follows: set $1=(\alpha=0.5, b=0.5, a=2), \quad$ set2 $=(\alpha=0.5, b=0.5, a=$ $3)$, set $3=(\alpha=0.3, b=0.5, a=2)$, and set $4=(\alpha=0.3, b=$ $0.5, a=3)$. The MLE, LSE, WLSE, CVE, PCE, MPSE, ADE, and RTADE of $\alpha, a$, and $b$ are determined. Then, the 
TABLE 7: Goodness of fit measures for the data sets.

\begin{tabular}{|c|c|c|c|c|c|}
\hline Model & $\ell$ & AIC & $\mathrm{BIC}$ & $A^{*}$ & $W^{*}$ \\
\hline \multicolumn{6}{|c|}{ Data I } \\
\hline APTIL & -216.492 & 438.984 & 444.597 & 0.27257 & 0.038885 \\
\hline APW & -225.272 & 456.544 & 462.158 & 1.52944 & 0.207808 \\
\hline APIE & -236.609 & 477.217 & 480.960 & 6.46851 & 1.221870 \\
\hline $\mathrm{APP}$ & -263.177 & 530.354 & 534.097 & 10.7680 & 2.139800 \\
\hline MOLBE & -224.746 & 453.492 & 457.235 & 1.55599 & 0.216626 \\
\hline IL & -235.826 & 475.652 & 479.394 & 6.48532 & 1.236730 \\
\hline \multicolumn{6}{|c|}{ Data II } \\
\hline APTIL & -197.035 & 400.070 & 405.684 & 0.265554 & 0.0345284 \\
\hline APIW & -198.597 & 403.195 & 408.809 & 0.539549 & 0.0651926 \\
\hline APIE & -200.564 & 405.129 & 408.871 & 0.857503 & 0.1104240 \\
\hline APP & -216.577 & 437.154 & 440.896 & 5.353100 & 0.9553700 \\
\hline MOLBE & -197.201 & 398.403 & 402.145 & 0.372636 & 0.0614331 \\
\hline IL & -205.056 & 414.112 & 417.854 & 2.530860 & 0.4221020 \\
\hline \multicolumn{6}{|c|}{ Data III } \\
\hline APTIL & -151.910 & 309.819 & 314.023 & 0.430672 & 0.056662 \\
\hline APIW & -153.147 & 312.293 & 316.497 & 0.491873 & 0.069053 \\
\hline APIE & -153.372 & 310.744 & 313.546 & 0.596184 & 0.089069 \\
\hline $\mathrm{APP}$ & -156.025 & 314.169 & 316.972 & 0.796196 & 0.108866 \\
\hline MOLBE & -155.336 & 314.673 & 317.475 & 2.259080 & 0.323858 \\
\hline IL & -153.639 & 310.279 & 315.081 & 0.458900 & 0.066987 \\
\hline
\end{tabular}

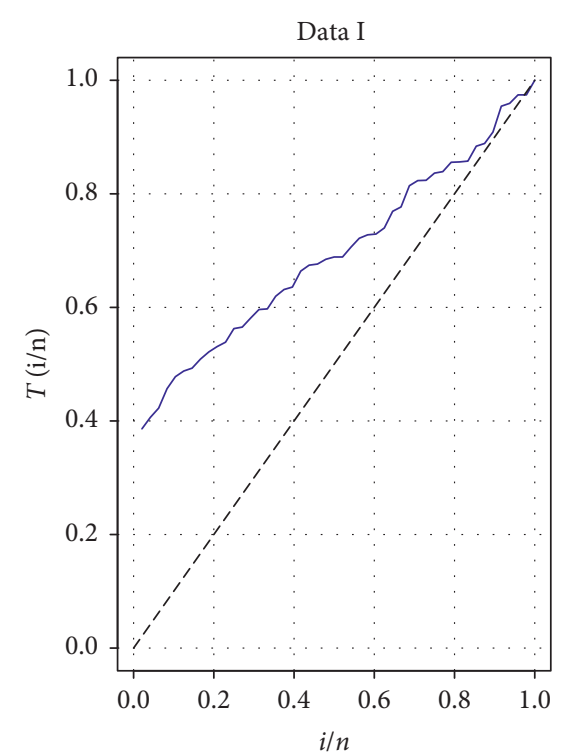

(a)

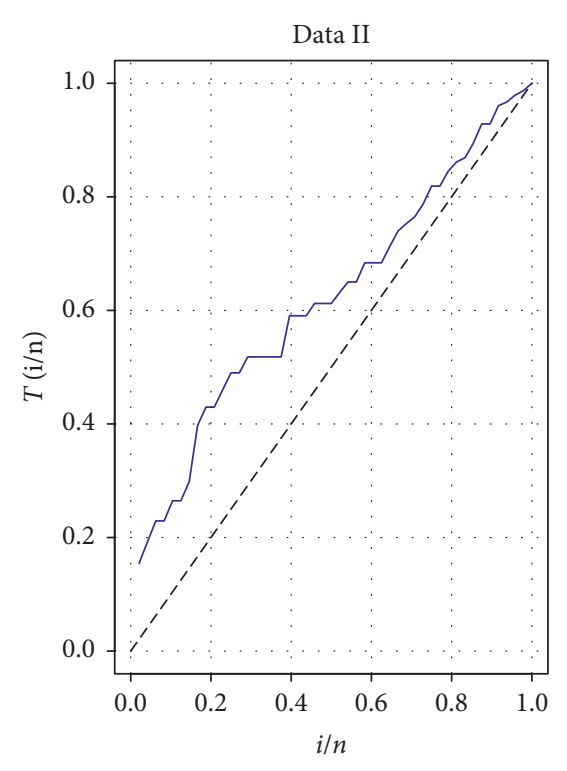

(b)

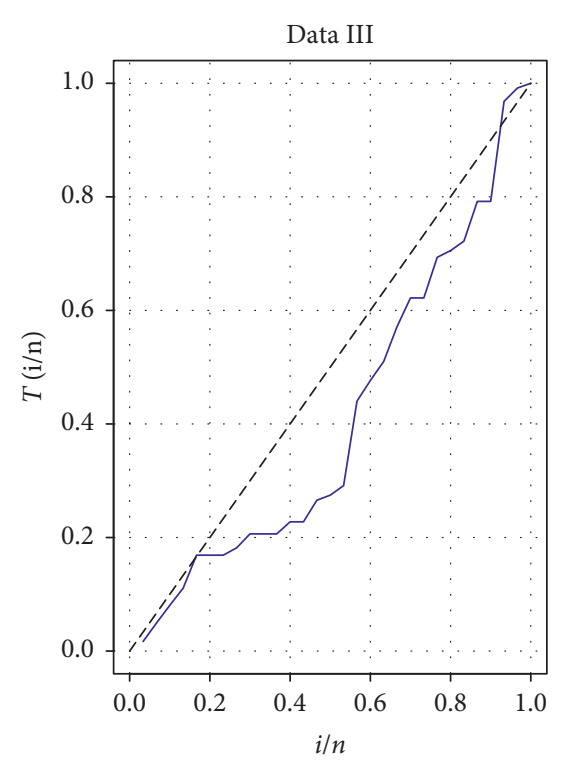

(c)

Figure 4: TTT Plots for all three data sets.

estimates of all methods and their mean square errors (MSEs) are documented in Tables 1-4.

Form Table 5, for the parameter combinations, we can conclude that the ML estimation method outperforms all the other estimation methods (overall score of 20.5). Therefore, depending on our study, we can consider the ML estimation method is the best for APTIL distribution.

\section{Applications}

In this section, we utilized three data sets to show that APTIL can be a better life testing distribution compared with some known probability distributions such as APT Weibull (APW) distribution [9], alpha power transformed inverse exponential (APTIE) distribution [10], 


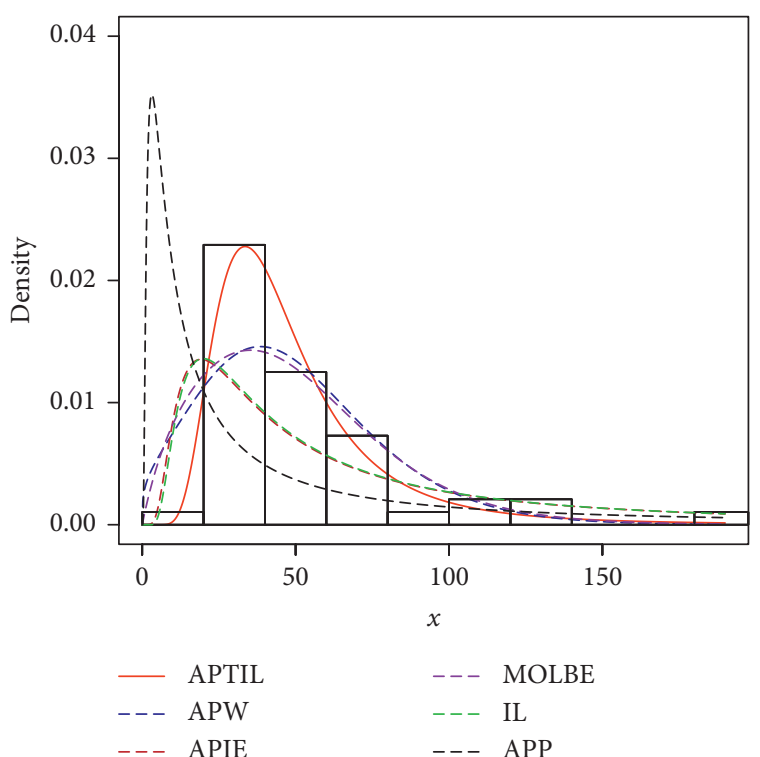

(a)

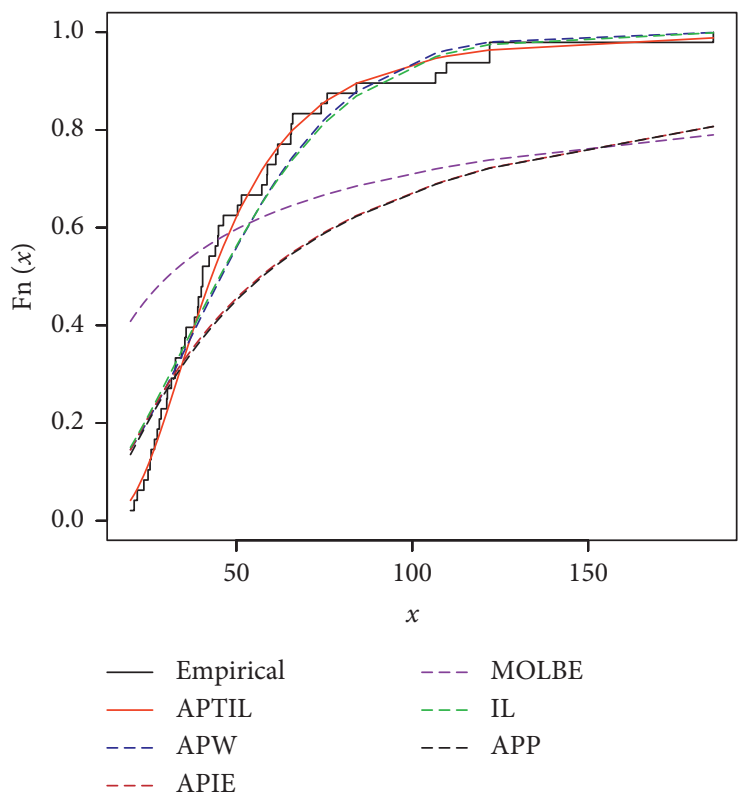

(b)

FIgURe 5: Estimated pdf and cdf of the APTIL model and other competing models for first data.

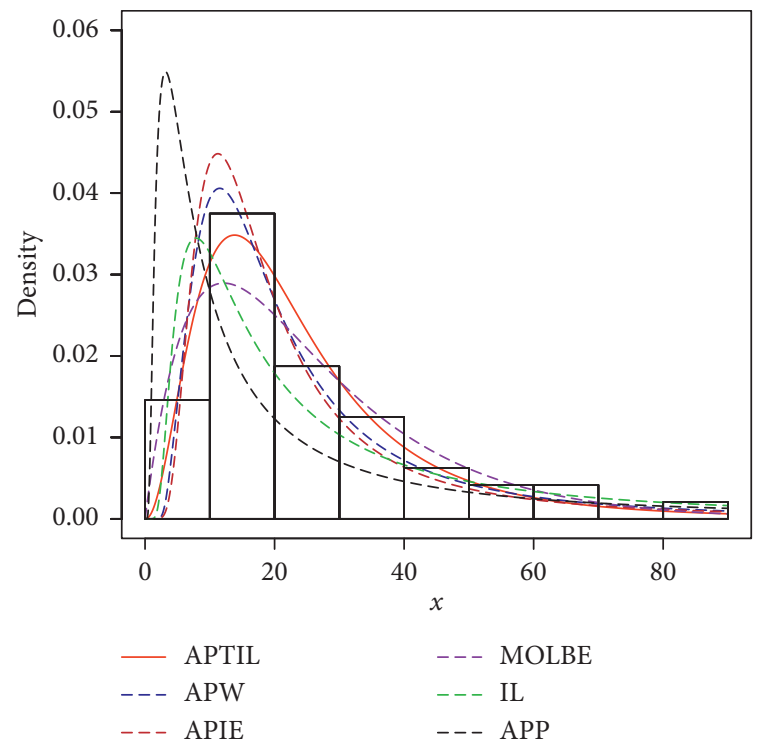

(a)

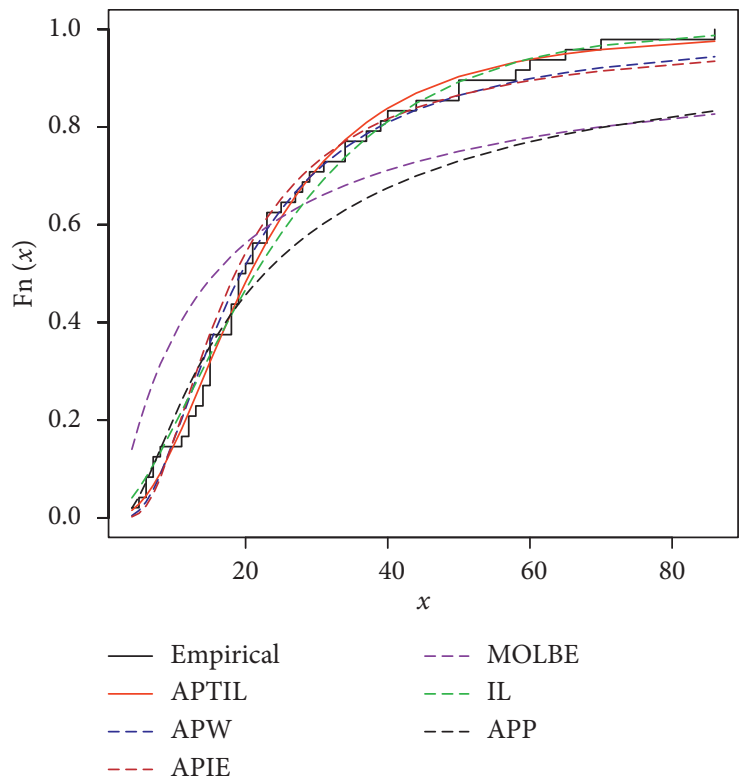

(b)

Figure 6: Estimated pdf and cdf of APTIL model and other competing models for second data.

Marshall Olkin length biased exponential (MOLBE) distribution [27], APT inverted Weibull (APIW) distribution [14], APT Pareto (APTP) distribution [17], and inverse Lomax (IL) distribution [4]. The corresponding probability density functions of competitor models are given below: 


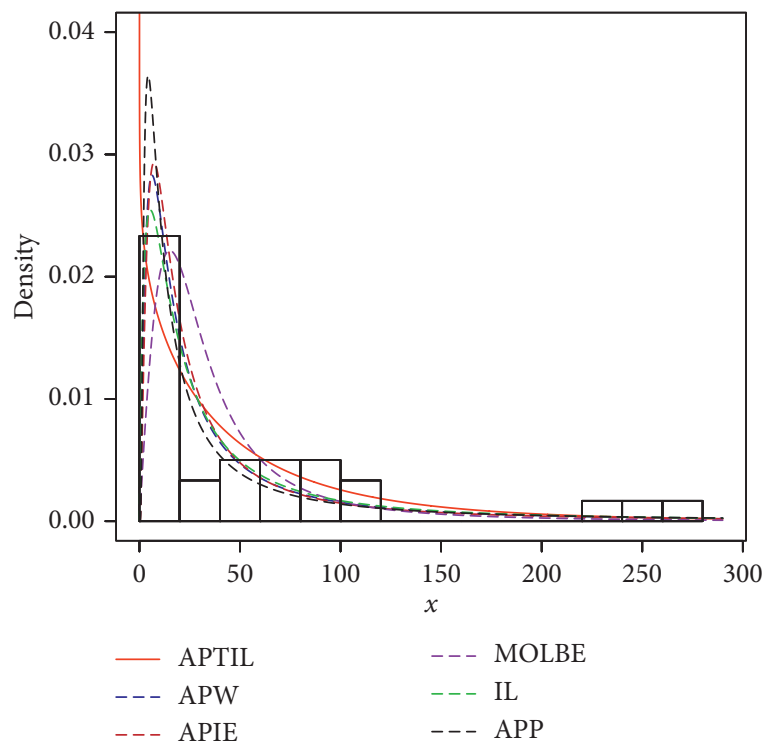

(a)

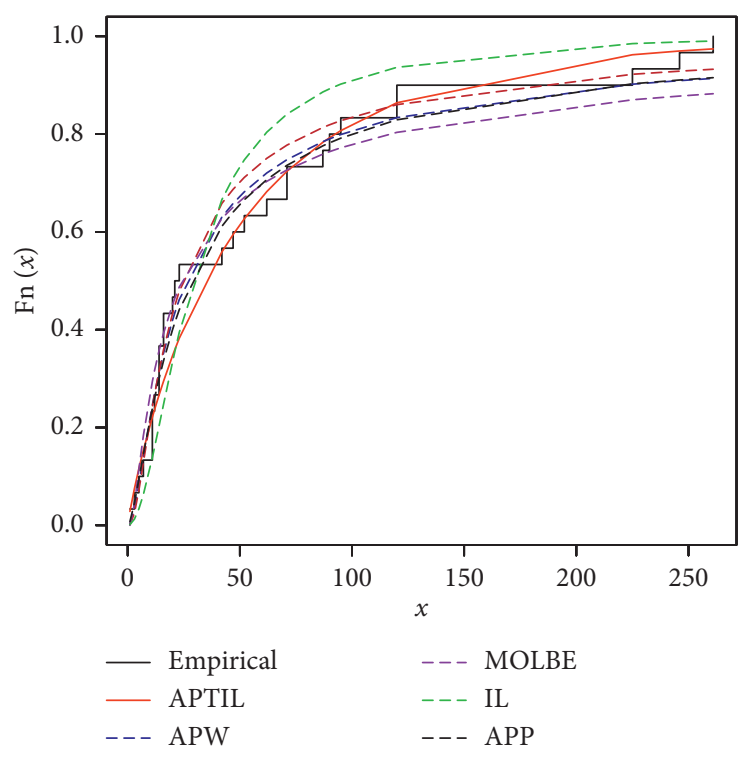

(b)

Figure 7: Estimated pdf and cdf of the APTIL model and other competing models for third data.

TABLE 8: Likelihood ratio test results for data sets.

\begin{tabular}{|c|c|c|c|}
\hline Model & Chi-square & $\mathrm{df}$ & $p$ value \\
\hline \multicolumn{4}{|c|}{ Data set I } \\
\hline APTIL vs APIE & 40.234 & 1 & $2.252929 e-10$ \\
\hline APTIL vs MOLBE & 16.508 & 1 & $4.844524 e-05$ \\
\hline APTIL vs APP & 93.370 & 1 & $4.337761 e-22$ \\
\hline APTIL vs IL & 38.668 & 1 & $5.023782 e-10$ \\
\hline \multicolumn{4}{|c|}{ Data set II } \\
\hline APTIL vs APIE & 7.0580 & 1 & 0.007891205 \\
\hline APTIL vs MOLBE & 2.3320 & 1 & 0.054483800 \\
\hline APTIL vs APP & 39.084 & 1 & $4.059573 e-10$ \\
\hline APTIL vs IL & 16.042 & 1 & $6.195283 e-05$ \\
\hline \multicolumn{4}{|c|}{ Data set III } \\
\hline APTIL vs APIE & 2.9240 & 1 & 0.05727128 \\
\hline APTIL vs MOLBE & 6.8520 & 1 & 0.00885421 \\
\hline APTIL vs APP & 8.2300 & 1 & 0.00412035 \\
\hline APTIL vs IL & 3.4580 & 1 & 0.05294641 \\
\hline
\end{tabular}

$$
\begin{aligned}
f_{\mathrm{APIE}}(x) & =\frac{\lambda \log (\alpha)}{\alpha-1} x^{2} e^{-(\lambda / x)} \alpha^{e^{-(\lambda / x)}}, \\
f_{\mathrm{APIW}}(x) & =\frac{\log (\alpha) \lambda \beta}{\alpha-1} x^{-(\beta+1)} e^{-\lambda x^{-\beta}} \alpha^{e^{-\lambda x^{-\beta}}}, \\
f_{\mathrm{APW}}(x) & =\frac{\log (\alpha) \lambda \beta}{\alpha-1} x^{(\beta-1)} e^{-\lambda x^{\beta}} \alpha^{1-e^{\lambda x^{\beta}}}, \\
f_{\mathrm{APP}}(x) & =\frac{\beta \log (\alpha)}{\alpha-1} x^{-(\beta+1)} \alpha^{1-e^{x^{-\beta}}}, \\
f_{\mathrm{MOLBE}}(x) & =\frac{\left(x / \beta^{2}\right) x e^{-(x / \beta)}}{\left(1-(1-\alpha)(1+(x / \beta)) e^{-(x / \beta)}\right)^{2}} .
\end{aligned}
$$

The first data set was originally reported by [28] which represents the maximum annual flood discharges of the North Saskachevan in unit of 1000 cubic feet per second, of the North Saskachevan River at Edmonton, over a period of 47 years. The data are as follows: $19.885,20.940,21.820$, 23.700, 24.888, 25.460, 25.760, 26.720, 27.500, 28.100, 28.600, $30.200,30.380,31.500,32.600,32.680,34.400,35.347,35.700$, 38.100, 39.020, 39.200, 40.000, 40.400, 40.400, 42.250, 44.020, $44.730,44.900,46.300,50.330,51.442,57.220,58.700,58.800$, $61.200,61.740,65.440,65.597,66.000,74.100,75.800,84.100$, $106.600,109.700,121.970,121.970$, and 185.560 .

The second data set represents the marks in Mathematics for 48 students in the slow pace programme in the year 2013 [29]. The data are as follows: $29,25,50,15,13,27,15,18,7,7$, $8,19,12,18,5,21,15,86,21,15,14,39,15,14,70,44,6,23$, 
$58,19,50,23,11,6,34,18,28,34,12,37,4,60,20,23,40,65$, 19 , and 31 .

The third data consists of a sample of 30 failure times of air-conditioned system of an airplane [30]. The data are as follows: $23,261,87,7,120,14,62,47,225,71,246,21,42,20$, $5,12,120,11,3,14,71,11,14,11,16,90,1,16,52$, and 95 .

For the selection of best fit model, we used the following criteria: Akaike information criterion (AIC), Bayesian information criterion (BIC), Anderson-darling (A*), and Cramer-von Mises $(\mathrm{W} *)$ test. The maximum likelihood estimates are presented in Table 6 , and the goodness of fit measures are presented in Table 7.

We can use the likelihood ratio (LR) test to compare the fit of the ALTIL distribution with other models for given data sets. The form of the test is suggested its name

$$
\text { LRT }=2 \log \left(\frac{L_{s}(\widehat{\theta})}{L_{g}(\widehat{\theta})}\right),
$$

where the LR is the ratio of two likelihood functions; the simpler model $(s)$ has fewer parameters than the general $(g)$ model. The LR test rejects the null hypothesis if $\chi^{2}>\chi_{d}^{2}$, where $\chi_{d}^{2}$ denotes the upper $100 \%$ point of the $\chi^{2}$ distribution.

The shape hazard function for modeling can be analyzed using graphical technique called total time in the test (TTT) plot (for more details, see [31]). From Figure 4, for the first and second data, the TTT plot is concave and provides evidence of the monotonic hazard rate. For the third data set, The TTT plot is convex and according to [31], it provides evidence that the hazard rate is decreasing.

The APTIL distribution gives the lowest values of AIC, BIC, $A^{*}$, and $W^{*}$ tests among all the fitted models to these data sets. So, it could be selected as the best model among them. The fitted pdf and estimated cumulative distribution function of the APTIL are displayed in Figures 5-7 for the three data sets, respectively.

The empirical data and estimated density plot show closeness which depict that the APTIL model fits all three data sets well. The APTIL model is compared with other competitive models. The estimated cdf curve of APTIL model also confirms the above results.

The likelihood ratio test is performed to compare APTIL distribution with other fitted models to test $H_{\mathrm{o}}$ against $H_{1}$ discussed above, and results are shown in Table 8. Low values of the likelihood ratio mean that the observed result was much less likely to occur under the null hypothesis as compared with the alternative. We conclude that APTIL distribution provides better fit than all other competitive models at the level of significance $\leq 0.05$.

\section{Conclusions}

In this research, we proposed and studied the APTIL distribution. Some structural characteristics of the APTIL distribution are derived. The asymptotic behavior of its density function is studied. Characterization related to hazard rate function is also obtained. Estimation of the population parameters is achieved using eight various procedures. Simulation results are carried out to assess the performance of estimators. Real data sets are used for the applications to show the flexibility of the APTIL model.

\section{Appendix}

\section{A. Proof of Lemma 2}

By using the series representation of exponential function and Taylor's series expansion of the function $\left(e^{\delta x}\right)$ in equation (27), we have

$$
\begin{aligned}
& \tau(a, b, \alpha, r, \delta)=\int_{0}^{\infty} x^{r-2} \sum_{i=0}^{\infty} \frac{(\ln \alpha)^{i}}{i !}\left(1+\frac{b}{x}\right)^{-a(i+1)-1} \sum_{j=0}^{\infty} \frac{(\delta x)^{j}}{j !} \mathrm{d} x, \\
& \tau(a, b, \alpha, r, \delta)=\sum_{i=0}^{\infty} \sum_{j=0}^{\infty} \frac{(\delta)^{j}}{j !} \frac{(\ln \alpha)^{i}}{i !} \int_{0}^{\infty} x^{r+a(i+1)+j-1}(b+x)^{-\{a(i+1)+1\}} \mathrm{d} x .
\end{aligned}
$$

Using the following relation given by [30],

$$
\begin{aligned}
\int_{0}^{\infty} x^{u-1}\left(p+q x^{v}\right)^{-(n+1)} \mathrm{d} x= & \frac{1}{v p^{n+1}}\left(\frac{p}{q}\right)^{u / v} \\
& \cdot \frac{\Gamma(u / v) \Gamma(1+n-(u / v))}{\Gamma(1+n)} \\
& \cdot\left[0<\frac{u}{v}<n+1, p \neq 0, q \neq 0\right], \\
\tau(a, b, \alpha, r, \delta)= & \sum_{i=0}^{\infty} \sum_{j=0}^{\infty} \frac{(\delta)^{j}}{j !} \frac{(\ln \alpha)^{i}}{i !}(b)^{r+j-1} \\
& \cdot \frac{\Gamma(r+a(i+1)+j) \Gamma(1-r-j)}{\Gamma(a(i+1)+1)} .
\end{aligned}
$$

\section{B. Code for Simulation}

$$
\begin{aligned}
u^{\langle j\rangle} & :=(\operatorname{runif}(n, 0,1)), \\
x_{i, j} & :=\frac{b 1}{\left[\sqrt[a 1]{(\log \alpha 1) /\left(\log \left[u_{i, j} \cdot(\alpha 1-1)+1\right]\right)}\right]-1}, \\
t^{\langle j\rangle} & :=\operatorname{sort}\left(x^{\langle j\rangle}\right) .
\end{aligned}
$$

For MLE and MPSE,

$$
\operatorname{SA} 1(j):=\operatorname{Maximize}(\log L 1, \alpha, a, b) .
$$

For other methods,

$$
\mathrm{YA} 2(j):=\operatorname{Minimize}(L 2, \alpha, a, b) \text {. }
$$

\section{Data Availability}

Data are given within the manuscript, and they are available in Section 6. 


\section{Conflicts of Interest}

The authors declare that they have no conflicts of interest.

\section{Acknowledgments}

This work was funded by the Deanship of Scientific Research (DSR), King Abdul Aziz University, Jeddah, under grant no. (DF-285-305-1441). The authors gratefully acknowledge the DSR technical and financial support.

\section{References}

[1] C. Kleiber and S. Kotz, Statistical Size Distributions in Economics and Actuarial Sciences, John Wiley \& Sons, Hoboken, NJ, USA, 2003.

[2] C. Kleiber, "Lorenz ordering of order statistics from log-logistic and related distributions," Journal of Statistical Planning and Inference, vol. 120, no. 1-2, pp. 13-19, 2004.

[3] D. McKenzie, C. Miller, and D. A. Falk, The Landscape Ecology of Fire, Springer Dordrecht Heidelberg, New York, NY, USA, 2011.

[4] S. K. Singh, U. Singh, and A. S. Yadav, "Reliability estimation for inverse Lomax distribution under type $\Pi$ censored data using Markov chain Monte Carlo method," International Journal of Mathematics and Statistics, vol. 17, no. 1, pp. 128-146, 2016.

[5] H. M. Reyad and S. A. Othman, "E-Bayesian estimation of two-component mixture of inverse Lomax distribution based on type-I censoring scheme," Journal of Advances in Mathematics and Computer Science, vol. 26, no. 2, pp. 1-22, 2018.

[6] A. S. Hassan and M. Abd-Allah, "On the inverse power Lomax distribution," Annals of Data Science, vol. 6, no. 2, pp. 259278, 2018.

[7] A. S. Hassan and R. E. Mohamed, "Weibull inverse Lomax distribution," Pakistan Journal of Statistics and Operation Research, vol. 15, no. 3, pp. 587-603, 2019.

[8] A. Mahdavi and D. Kundu, "A new method for generating distributions with an application to exponential distribution," Communications in Statistics-Theory and Methods, vol. 46, no. 13, pp. 6543-6557, 2017.

[9] S. Dey, V. K. Sharma, and M. Mesfioui, "A new extension of Weibull distribution with application to lifetime data," Annals of Data Science, vol. 4, no. 1, pp. 31-61, 2017.

[10] S. Dey, A. Alzaatreh, C. Zhang, and D. Kumar, "A new extension of generalized exponential distribution with application to ozone data," Ozone: Science \& Engineering, vol. 39, no. 4, pp. 273-285, 2017.

[11] S. Dey, I. Ghosh, and D. Kumar, "Alpha-power transformed Lindley distribution: properties and associated inference with application to earthquake data," Annals of Data Science, vol. 6, no. 4, pp. 623-650, 2019.

[12] A. S. Hassan, R. E. Mohamd, M. Elgarhy, and A. Fayomi, "Alpha power transformed extended exponential distribution: properties and applications," Journal of Nonlinear Sciences and Applications, vol. 12, no. 4, pp. 62-67, 2018.

[13] C. Unal, S. Cakmakyapan, and G. Ozel, "Alpha power inverted exponential distribution: properties and application," Gazi University Journal of Science, vol. 31, no. 3, pp. 954-965, 2018.

[14] D. A. and W. Magdy, "On the alpha-power inverse Weibull distribution," International Journal of Computer Applications, vol. 181, no. 11, pp. 6-12, 2018.

[15] S. Dey, M. Nassar, and D. Kumar, "Alpha power transformed inverse Lindley distribution: a distribution with an upside- down bathtub-shaped hazard function," Journal of Computational and Applied Mathematics, vol. 348, pp. 130-145, 2019.

[16] A. S. Hassan, M. Elgarhy, R. E. Mohamd, and S. Alrajhi, "On the alpha power transformed power Lindley distribution," Journal of Probability and Statistics, vol. 2019, Article ID 8024769, 13 pages, 2019.

[17] S. Ihtisham, A. Khalil, S. Manzoor, S. A. Khan, and A. Ali, "Alpha-power Pareto distribution: its properties and applications," PLoS One, vol. 14, no. 6, 2019.

[18] B. Fisher and A. Kilicman, "Some results on the gamma function for negative integers," Applied Mathematics \& Information Sciences, vol. 6, no. 2, pp. 173-176, 2012.

[19] J. J. Swain, S. Venkatraman, and J. R. Wilson, "Least-squares estimation of distribution functions in Johnson's translation system," Journal of Statistical Computation and Simulation, vol. 29, no. 4, pp. 271-297, 1988.

[20] J. H. Kao, "Computer methods for estimating Weibull parameters in reliability studies," IRE Transactions on Reliability and Quality Control, vol. 13, pp. 15-22, 1958.

[21] J. H. K. Kao, "A graphical estimation of mixed Weibull parameters in life-testing of electron tubes," Technometrics, vol. 1, no. 4, pp. 389-407, 1959.

[22] R. D’Agostino and M. Stephens, Goodness-of-Fit Techniques, Marcel Dekker, New York, NY, USA, 1986.

[23] A. Luceño, "Fitting the generalized Pareto distribution to data using maximum goodness-of-fit estimators," Computational Statistics \& Data Analysis, vol. 51, no. 2, pp. 904-917, 2006.

[24] P. D. M. Macdonald, "Comments and queries comment on "an estimation procedure for mixtures of distributions" by choi and bulgren," Journal of the Royal Statistical Society: Series B (Methodological), vol. 33, no. 2, pp. 326-329, 1971.

[25] R. C. H. Cheng and N. A. K. Amin, "Maximum product of spacings estimation with application to the lognormal distributions," Math Report 79-1, Department of Mathematics, UWIST, Cardiff, UK, 1979.

[26] T. W. Anderson and D. A. Darling, "Asymptotic theory of certain "goodness of fit" criteria based on stochastic processes," The Annals of Mathematical Statistics, vol. 23, no. 2, pp. 193-212, 1952.

[27] M. A. U. Haq, R. M. Usman, S. Hashmi, and A. I. Al-Omeri, "The Marshall-Olkin length-biased exponential distribution and its applications," Journal of King Saud University-Science, vol. 31, no. 2, pp. 246-251, 2019.

[28] R. D. Gupta and D. Kundu, "A new class of weighted exponential distributions," Statistics, vol. 43, no. 6, pp. 621-634, 2009.

[29] H. Linhart and W. Zucchini, Model Selection, Wiley, New York, NY, USA, 1986.

[30] M. V. Aarset, "How to identify a bathtub hazard rate," IEEE Transactions on Reliability, vol. 36, no. 1, pp. 106-108, 1987.

[31] M. A. Montfort, "On testing that the distribution of extremes is of type I when type II is the alternative," Journal of $\mathrm{Hy}$ drology, vol. 11, no. 4, pp. 421-427, 1970. 